\title{
Rare Occurrence of Chemotherapy-Induced Regression of Focal Nodular Hyperplasia: A Case Report and Literature Review Johara AlMulhim \\ ${ }^{1}$ Department of Radiology, College of Medicine, King Faisal University, Al-Ahsa, Saudi Arabia Corresponding author: Johara AlMulhim, E-mail: Dr.johara1988@gmail.com
}

\begin{abstract}
Background: Chemotherapy exerts a multitude of effects on all organ systems including the liver. Chemotherapyinduced liver changes are widely discussed in the literature and include hepatitis, hepatic steatosis, cholestasis, nodular hyperplasia, and veno-occlusive disease among others. The exact mechanism of which nodular hyperplasia is affected by chemotherapy is controversial but some experts suggest an alteration in blood supply might lead to exaggerated focal regeneration.

Objectives: This study aimed to discuss an unusual effect of chemotherapeutic agents on pre-existing focal nodular hyperplasia with a short literature review.

Patient and methods: A case report of 67 years old bedridden male patient presenting to the hospital with a 1month history of gross hematuria who was treated with chemotherapy.

Results: Our case showed an interval size reduction of a pre-treatment documented FNH in a patient with muscleinvasive urinary bladder cancer who was treated with a chemotherapy regimen of GemCarbo. This report also emphasizes the importance of enhanced MRI with hepatobiliary contrast agents in the evaluation of equivocal liver lesions in cancer patients to avoid misdiagnosis of distant metastasis.

Conclusion: FNH is a common focal hepatic lesion and frequently seen in oncology patients during either initial evaluation or at follow up. Diagnostic radiologists should be aware of the expected imaging findings of various types of hepatic lesions and the effect of the medications the patient is receiving to avoid over-staging oncology patients.
\end{abstract}

Key words: Chemotherapy, FNH, MRI.

\section{INTRODUCTION}

The idea of chemotherapy for the treatment of various types of cancers first came to light during the early 20th century ${ }^{[1]}$. The first line of chemotherapeutic agents was generally cytotoxic. Thereafter, multiple chemotherapeutic agents have evolved with multiple mechanisms of actions including cytotoxic agents and those that affect the cell on molecular level interfering and altering the programmed cell growth, nutritional supply, and cell differentiation ${ }^{[2]}$. Chemotherapeutic agents exert innumerable effects all-over the human body through different pathways.

The presence of pre-existing focal liver lesions in newly diagnosed cancer patients is not uncommon. Indeed, as focal nodular hyperplasia ranks second in the list of common benign hepatic lesions, preceded only by hemangiomas [3]. The effect of medications, including chemotherapeutic agents, on these benign lesions, is not frequently reported.

In this report, we will discuss an unusual effect of chemotherapeutic agents on pre-existing focal nodular hyperplasia with a short literature review.

\section{Ethics approval and consent to publication}

Ethics approval for case reports is not required by the IRB. Written informed consent was obtained from the patients for this publication.

\section{CASE REPORT}

We report a 67 years old bedridden male patient presenting to the hospital with a 1-month history of gross hematuria. Upon evaluation, the patient was vitally stable with a normal abdominal physical examination. Upon reviewing his laboratory results, the only abnormalities were anemia $(\mathrm{Hg}: 10.9 \mathrm{~g} / \mathrm{dL})$ and positive urine RBCs (250/hpf). The treating urologist ordered an enhanced CT scan of the abdomen and pelvis which showed a large urinary bladder base fungating, partially calcified mass invading the urinary bladder neck and prostate (Figure 1). No synchronous urothelial cancer was detected. However, there was a sizable right hepatic lobe arterially enhancing lesion without significant washout (Figure 2).

The reporting radiologist raised the possibility of the hepatic lesion being focal nodular hyperplasia $(\mathrm{FNH})$ rather than a genuine metastasis and ordered an enhanced liver magnetic resonant imaging (MRI) using a hepatobiliary specific agent (Primovist). the lesion demonstrated slightly high signal intensity on T2 weighted images, arterial hyperenhancement and contrast uptake in the hepatobiliary phase consistent with the presumed diagnosis of FNH (Figure 3). No other hepatic lesions were seen.

The patient underwent a trial of cystoprostatectomy but failed due to significant pelvis adhesions. A cystoscopic biopsy confirmed the presence of muscle-invasive bladder cancer and, therefore, the patient was started on chemotherapy course of Gemcitabine and Carboplatin (GemCarbo). After completion of three cycles, a reevaluationenhanced CT scan of the abdomen and pelvis was performed showing a good response of the bladder tumor with post-chemotherapy changes (Figure 4), 
however, there was size reduction of the developed hepatic lesions or abdominopelvic aforementioned hepatic lesion without newly metastasis (Figure 5).

\section{FIGURES}

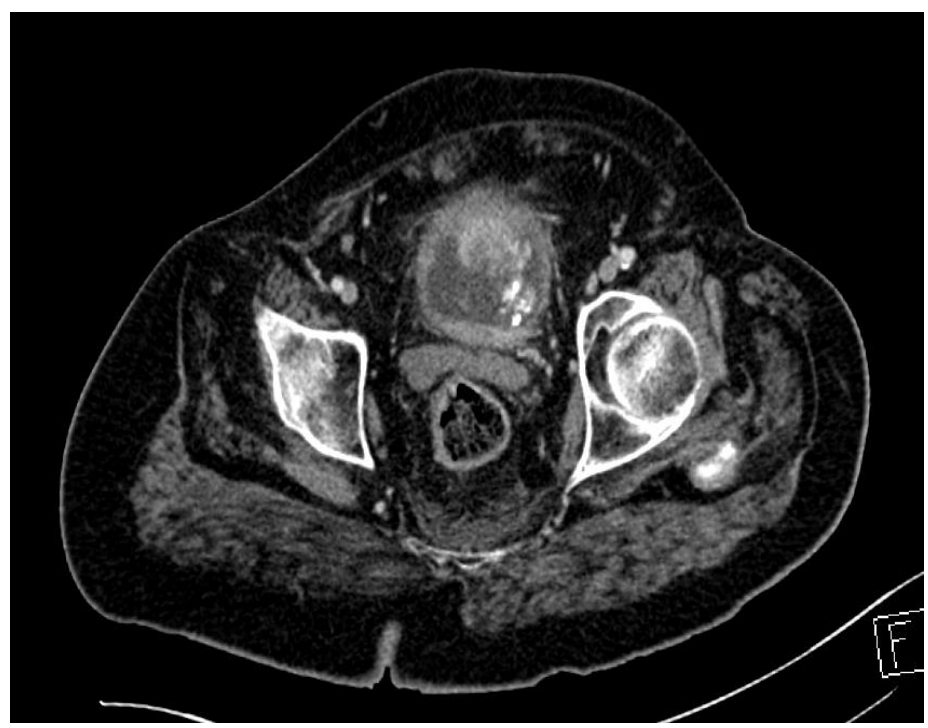

Figure (1): Axial image of contrast-enhanced CT scan of the abdomen and pelvis at the level of the mid urinary bladder during venous phase showing anterior urinary bladder wall thickening with endoluminal fungating mass containing calcifications consistent with biopsy proven muscle invasive urothelial carcinoma.

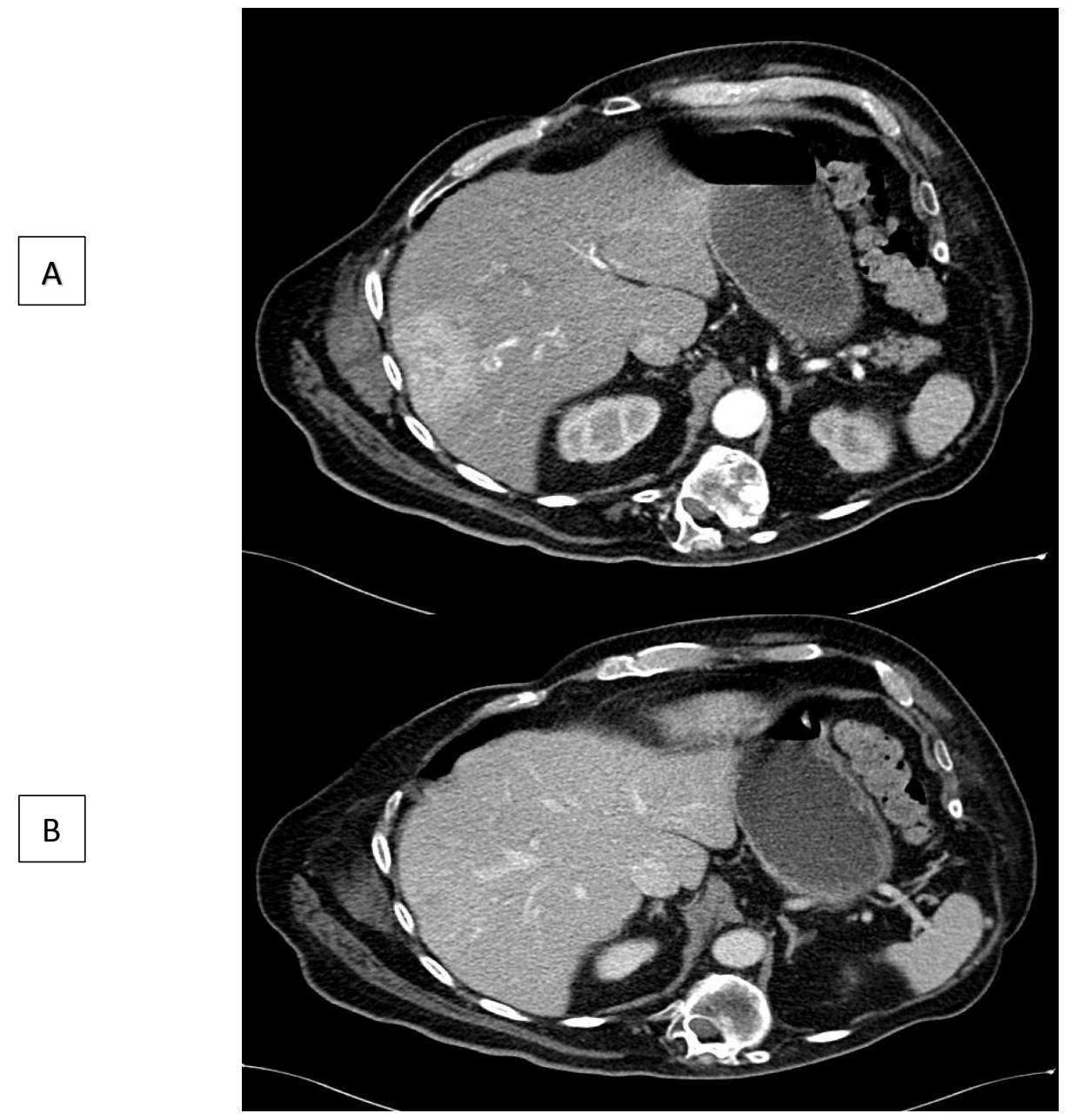


Figure (2): Axial images of enhanced CT scan of the abdomen and pelvis at the level of the liver. At arterial phase (A), there was a well-defined subcapsular right hepatic lobe avidly enhancing lesion. At venous phase (B), no significant washout was seen.
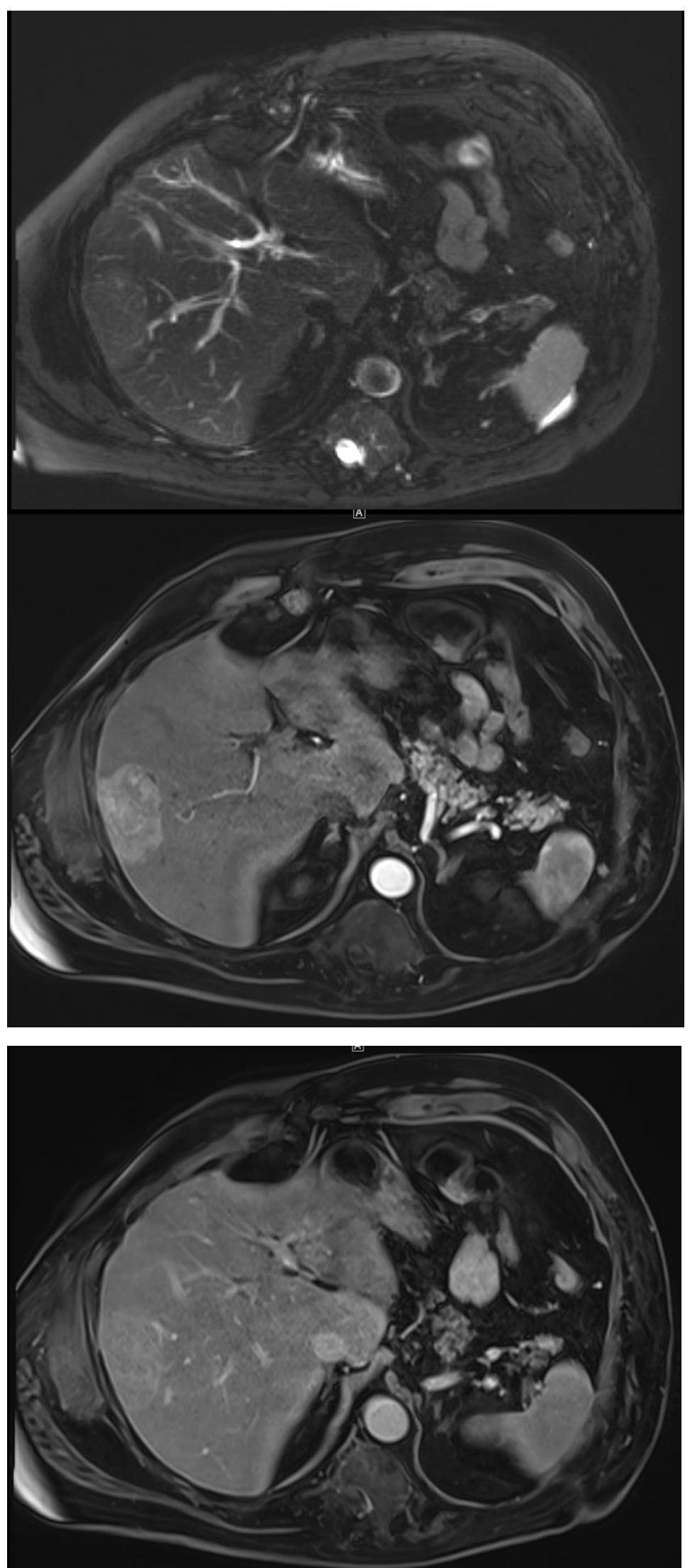


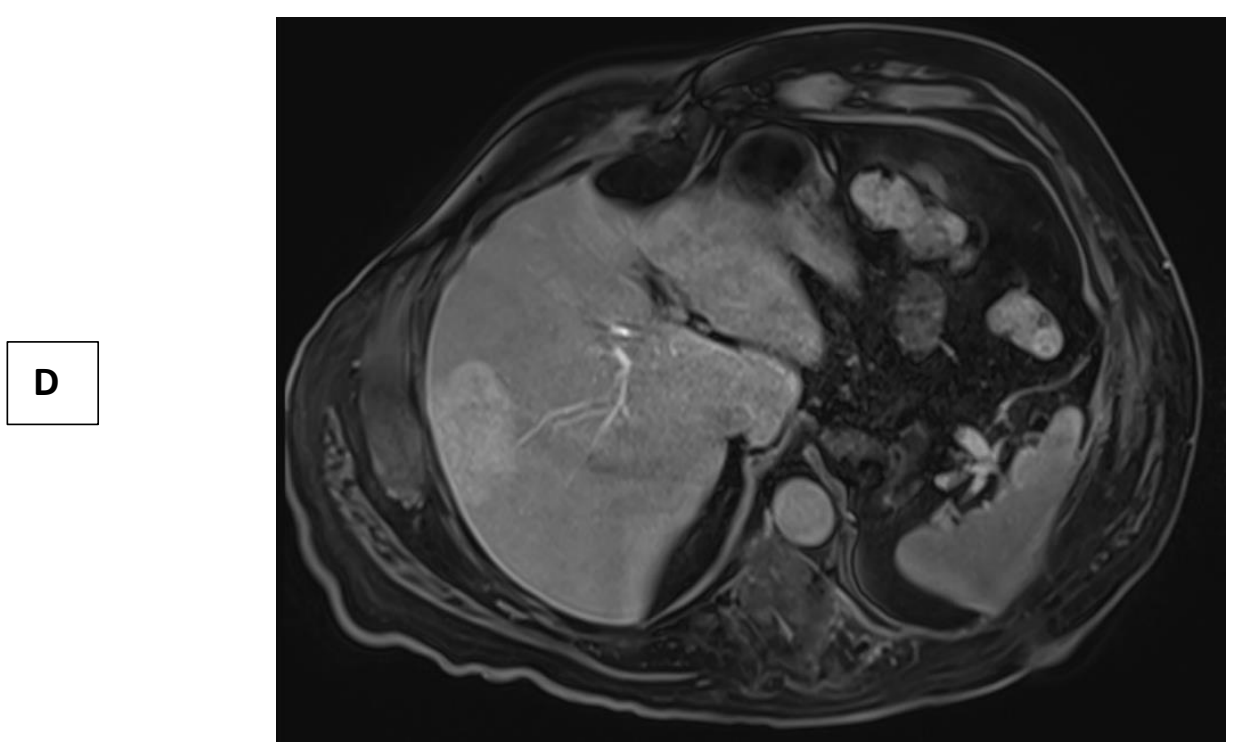

Figure (3): Axial images of contrast-enhanced MRI of the liver with hepatobiliary specific agent (Primovist). T2 weighted image (A) showing mildly high signal intensity of the subcapsular right hepatic lobe lesion. T1 post contrast images during arterial (B), equilibrium (C) and 25 minutes delay (hepatobiliary) phase(D) showing avid arterial enhancement of the lesion which becomes less prominent in the equilibrium phase with contrast accumulation in the hepatobiliary phase confirming the diagnosis of a focal nodular hyperplasia. Also, note the accumulation of contrast in the intra-hepatic bile ducts in the hepatobiliary phase.

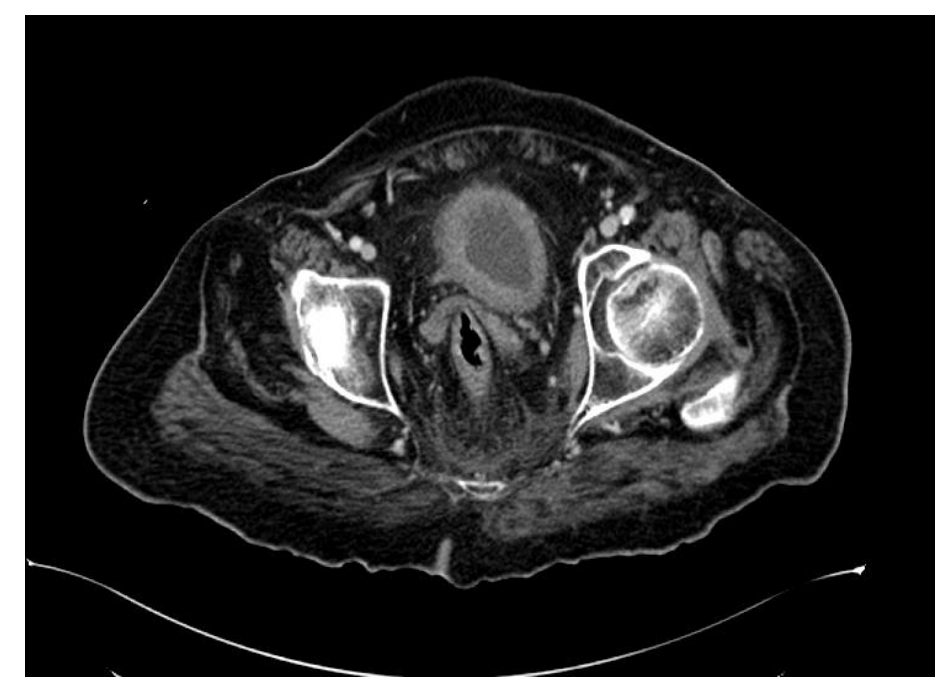

Figure (4): Axial images of enhanced CT scan of the abdomen and pelvis during venous phase after 3 cycles of GemCarbo. There was significant interval improvement of the fungating urinary bladder lesion with diffuse urinary bladder wall thickening, likely secondary to post treatment changes. 


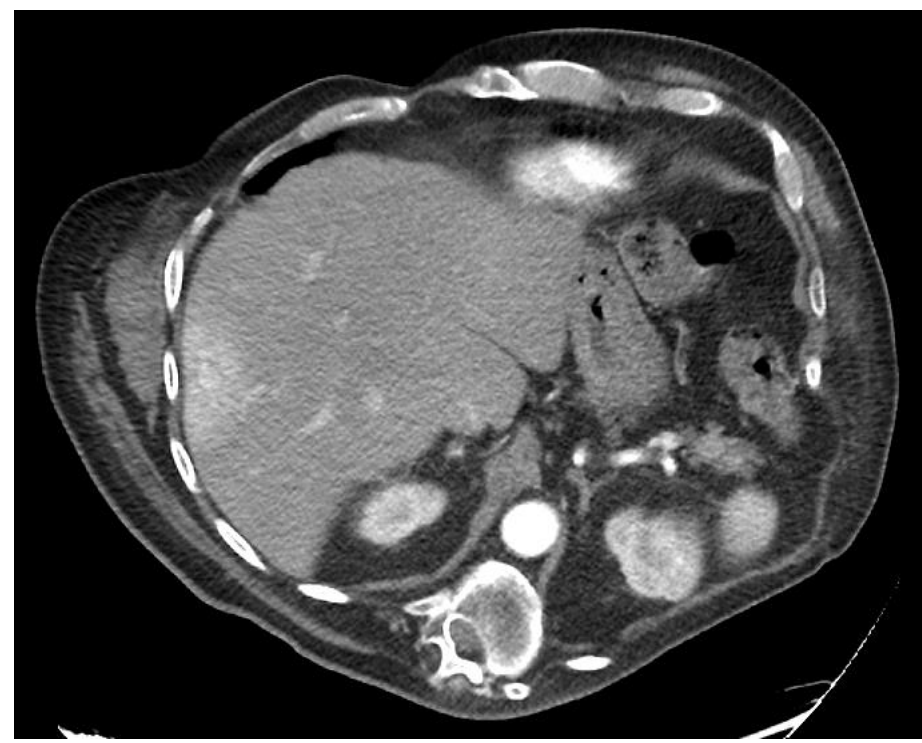

Figure (5): Axial images of enhanced CT scan of the abdomen and pelvis during arterial phase after 3 cycles of GemCarbo. Here we appreciated a regression in size of the known right hepatic lobe FNH. No newly developed lesions.

\section{DISCUSSION}

Chemotherapeutic agents that are currently available for solid tumors, including urinary bladder cancer, can be used before surgery and are termed neoadjuvant chemotherapy or after surgery in which case they are called adjuvant chemotherapy.

There are multiple classes of chemotherapeutic agents, including but not limited to alkylating agents, anti-metabolites, anti-tumor antibiotics, isomerase inhibitors, mitotic inhibitors, immunotherapy, hormonotherapy and steroids amongst others ${ }^{[2]}$. Each class of chemotherapeutic agents can induce a cascade of liver changes by various methods as the liver is the primary metabolism site of those medications, therefore, meticulous evaluation of the patient's pretherapeutic hepatic function tests and close follow up after initiation of chemotherapy is advised ${ }^{[4]}$.

The presentation of chemotherapy-induced liver injury is widely variable. Some patients would be completely asymptomatic with abnormalities only detected during evaluation of liver function tests while others can present with right upper quadrant pain, jaundice, itching, signs of volume overload and even hepatic encephalopathy ${ }^{[4]}$. Chemotherapy-induced liver changes include acute hepatitis, intrahepatic cholestasis, hepatic steatosis, biliary strictures as well as veno-occlusive disease, which have been widely reported ${ }^{[2,4]}$. Also, the development of new benign hepatic lesions or fibrosis has been seen in practice resulting in $\mathrm{FNH}$ and pseudocirrhosis respectively ${ }^{[4]}$. The exact mechanism of development of FNHs in those patients remains controversial but some experts suggest that alteration in the arterial supply by the medications might ignite a cascade of parenchymal changes leading to regenerative hyperplasia. Multiple articles have discussed the development of FNH in pediatric patients, which is a rare occurrence in the general population, after chemotherapy for neuroblastoma or rhabdomyosarcoma ${ }^{[5]}$. In particular, development of focal nodular hyperplasia after therapy with carboplatin has been reported in the literature, for example, Junewick et al. ${ }^{[6]}$ reported development of multiple FNHs after the patient was treated with a course of carboplatin, adriamycin, cyclophosphamide, cisplatin, vincristine, and etoposide chemotherapy, focal irradiation and stem cell bone marrow transplantation.

In general, fluctuation in the size of FNH is a documented phenomenon, however, near or total resolution is rarely reported most of which occurred after cessation of the oral contraceptive pill (OCP) or anabolic steroid usage although the exact pathophysiological mechanism of which hormones affect the course of FNH is not completely understood ${ }^{[7]}$.

Mamone et al. ${ }^{[8]}$ reported a case of a 26 years old female who presented with a giant FNH and 5 years history of OCP use that was followed up after 7 years with a total resolution of the FNH after discontinuation of OCP. Sarma et al. ${ }^{[3]}$ also reported 2 cases of near-total resolution of FNHs after discontinuation of $\mathrm{OCP}$ and exogenous estrogen. 
Interestingly, data also exists on interval size reduction of FNH after hormonotherapy for breast cancer. Cesario et al. ${ }^{[7]}$ reported a case of a 40 years old woman with breast cancer who was found to have FNH at initial diagnosis with significant size reduction over the course of 5 years after receiving local therapy for her primary tumor and a course of a synthetic analog of gonadotropin-releasing hormone (antiestrogenic).

There have been, up to the author's best knowledge, no reported cases of interval reduction in the size of pre-existing FNH after initiation of chemotherapy. Our case showed an interval size reduction of a pre-treatment documented FNH in a patient with muscle-invasive urinary bladder cancer who was treated with a chemotherapy regimen of GemCarbo. This report also emphasizes the importance of enhanced MRI with hepatobiliary contrast agents in the evaluation of equivocal liver lesions in cancer patients to avoid misdiagnosis of distant metastasis.

\section{CONCLUSION}

FNH is a common focal hepatic lesion and frequently seen in oncology patients during either initial evaluation or at follow up. Diagnostic radiologists should be aware of the expected imaging findings of various types of hepatic lesions and the effect of the medications the patient is receiving to avoid over-staging oncology patients.

\section{ACKNOWLEDGMENTS}

Author has nothing to acknowledge.

\section{REFERENCES}

1.DeVita V, Chu E (2008): A History of Cancer Chemotherapy. Cancer Research, 68 (21): 8643.

2. Sharma A, Houshyar R, Bhosale $P$ et al. (2014): Chemotherapy induced liver abnormalities: an imaging perspective. Clinical and molecular hepatology, 20 (3): 317-326.

3. Sarma A, Shaaban A, Heilbrun M et al. (2015): Two cases of near-complete regression of focal nodular hyperplasia of the liver: Case reports and review of the literature. Radiology case reports, 7 (3): 681-681.

4. Grigorian A, O'Brien C (2014): Hepatotoxicity Secondary to Chemotherapy. Journal of clinical and translational hepatology, 2 (2): 95-102.

5. Tugcu D, Kebudi R, Akici F et al. (2014): Hepatic Focal Nodular Hyperplasia Developing After Childhood Cancers: Two-Center's Experience from Turkey. UhodUluslararasi Hematoloji-Onkoloji Dergisi., 24: 112-117.

6. Junewick J, Mitchell D (2006): Focal nodular hyperplasia in oncology patients. Pediatric radiology, 36: 464.

7. Cesario V, Riccardi L, Giovinale M et al. (2014): Regression of focal nodular hyperplasia after hormonotherapy: A case report. Digestive and Liver Disease, 46: S116.

8. Mamone G, Caruso S, Cortis K et al. (2016): Complete spontaneous regression of giant focal nodular hyperplasia of the liver: Magnetic resonance imaging evaluation with hepatobiliary contrast media. World journal of gastroenterology, 22 (47): 10461-10464. 